\title{
Comparison of the Clinical Characteristics of Pneumocystis Pneumonia between Patients with Rheumatoid Arthritis Being Treated with Biologics and Those Being Treated without Biologics
}

\author{
Mitsuhiro Akiyama, Yuko Kaneko, and Tsutomu Takeuchi \\ Division of Rheumatology, Department of Internal Medicine, Keio University School of Medicine, Tokyo, Japan \\ Correspondence should be addressed to Yuko Kaneko; ykaneko@z6.keio.jp
}

Received 30 January 2017; Accepted 11 June 2017; Published 12 July 2017

Academic Editor: Keiju Hiromura

Copyright (C) 2017 Mitsuhiro Akiyama et al. This is an open access article distributed under the Creative Commons Attribution License, which permits unrestricted use, distribution, and reproduction in any medium, provided the original work is properly cited.

\begin{abstract}
Objective. The aim of this study was to compare the clinical characteristics of pneumocystis pneumonia (PCP) between patients with rheumatoid arthritis (RA) being treated with biologics and those being treated without biologics. Methods. From 8,630 patients with RA in our institution, we enrolled 24 patients who had developed PCP during the course of their treatment. They were divided into two groups according to the treatment they were receiving for RA: the biologics group $(n=12)$ and the nonbiologics group $(n=12)$. Clinical characteristics of PCP were compared between the two groups. Results. At PCP diagnosis, the biologics group showed significantly lower serum levels of $\beta$-D-glucan and C-reactive protein than the nonbiologics group, while the biologics group had significantly higher lymphocyte counts than the nonbiologics group. In the nonbiologics group, lower lymphocyte counts were associated with higher $\beta$-D-glucan levels; however, this was not observed in the biologics group. Conclusion. The finding that RA patients being treated with biologics developed PCP with relatively normal lymphocyte counts and lower $\beta$-D-glucan levels suggests that the pathophysiology of PCP in those patients is different from that in patients being treated with other antirheumatic drugs.
\end{abstract}

\section{Introduction}

Pneumocystis pneumonia (PCP) is a rare but critical complication in immunosuppressed patients [1]. It was initially discovered in human immunodeficiency virus (HIV) positive patients in the 1980s [2]. In the following years, PCP cases increased in patients with rheumatoid arthritis (RA) being treated with disease-modifying antirheumatic drugs (DMARDs), with high mortality rates recorded $[3,4]$. More recently, PCP cases have also been reported during treatment with biologic agents targeted at specific molecules $[5,6]$.

While immunosuppressive therapy is identified as a risk factor for the development of PCP, through lymphocyte count suppression [7], a previous study reported that PCP could develop during treatment with biologic agents in patients with RA, without apparent lymphocytopenia [8].
This suggests that distinct mechanisms may be involved in the development of PCP during treatment with biologic agents. However, little is known about the differences in the clinical characteristics of PCP during treatment with biologic agents in comparison with nonbiologic DMARDs.

The objective of this study was to elucidate the differences in the clinical characteristics of PCP between patients being treated with biologic agents and patients being treated with nonbiologic DMARDs.

\section{Patients and Methods}

2.1. Study Design. The study was a retrospective, observational clinical study conducted at our institution with approval by the institutional ethics committee. Written informed consent was waived based on Japanese guidelines. 
From a total of 8,630 consecutive patients with RA who visited our institution between March 1999 and September 2016, all patients who were diagnosed with PCP were enrolled in the analysis.

PCP was diagnosed as definite when patients met all the following criteria [9]: (a) clinical manifestations and findings compatible with PCP on chest computed tomography (CT), (b) microscopic detection of Pneumocystis jirovecii or positive polymerase chain reaction test results for Pneumocystis jirovecii DNA in respiratory specimens, (c) increased $\beta$ D-glucan serum levels. PCP was diagnosed as probable when patients met criterion (a), with either criterion (b) or (c) in addition [9]. The manufacturer suggested that the upper normal limit for serum $\beta$-D-glucan was $11.3 \mathrm{pg} / \mathrm{mL}$ (Wako Pure Chemical Industries, Tokyo, Japan), but we defined $31.1 \mathrm{pg} / \mathrm{mL}$ as a cut-off value in this study to exclude Pneumocystis jirovecii colonization, based on a previous study [10].

2.2. Clinical Assessments. Demographic characteristics (age, sex, smoking status, and duration from onset of symptoms to PCP diagnosis), comorbidities, associated medical conditions, and laboratory findings (white blood cell count, lymphocyte count, lactate dehydrogenase [LDH], C-reactive protein $[\mathrm{CRP}]$, immunoglobulin $\mathrm{G}$ [IgG], Krebs von den Lungen-6 [KL-6], and $\beta$-D-glucan) were retrospectively reviewed. The severity of pneumonia was evaluated with the oxygenation index determined from the arterial oxygen tension and the fraction of inspired oxygen $\left(\mathrm{PaO}_{2} / \mathrm{FiO}_{2}\right.$ ratio).

2.3. Statistical Analysis. Continuous variables were expressed as mean \pm standard error. Mann-Whitney $U$ test and Fisher's exact test were used to compare continuous and categorical variables, respectively. The relationships between variables were analysed by Spearman correlation coefficient. SPSS version 22.0 (IBM, Armonk, NY, USA) was used for all statistical analyses, and $P<0.05$ was considered as significant.

\section{Results}

3.1. Recruitment of Patients. Figure 1 is an outline showing how patients were recruited for this study. Of the 8,630 patients with RA, 1712 patients received biologics. Thirtythree patients were suspected to have PCP. Of these 33 patients, 9 patients were excluded from the analysis because they did not satisfy the diagnostic criteria stated above. Consequently, a total of 24 patients were enrolled in this study (definite PCP, $n=16$, and probable PCP, $n=8$ ) and divided into two groups according to their treatment for RA (the biologics group, $n=12$ (definite, $n=7$ and probable, $n=5$ ), and the nonbiologics group, $n=12$ (definite, $n=9$ and probable, $n=3)$ ). Patients in the biologics group received infliximab, $n=7$; adalimumab, $n=2$; etanercept, $n=1$; golimumab, $n=1$; and abatacept, $n=1$.

3.2. Comparison between Patients Being Treated with Biologics and Those Being Treated with Nonbiologics. Baseline demographic characteristics of the patients at the time of their

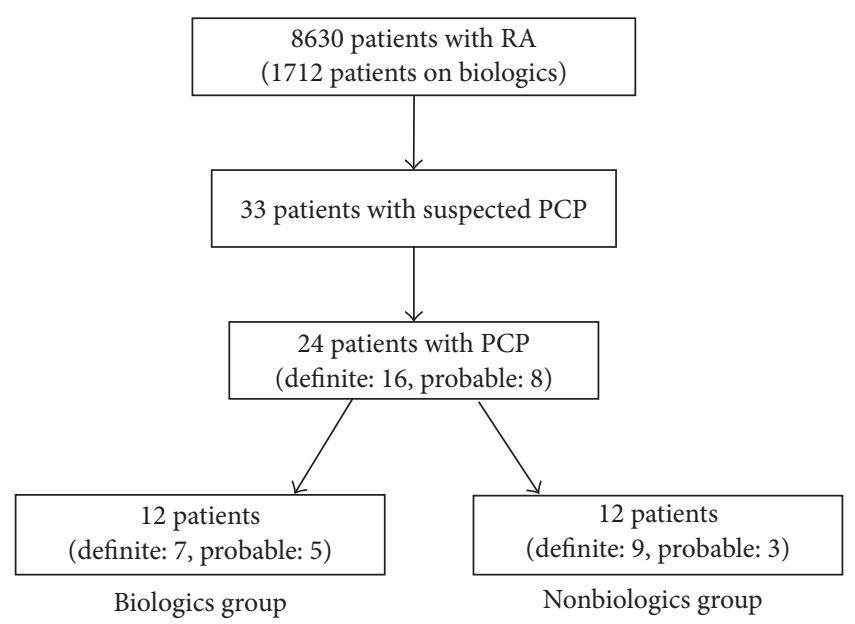

FIGURE 1: Flowchart of inclusion of patients. RA, rheumatoid arthritis. PCP, pneumocystis pneumonia.

diagnosis of PCP are shown in Table 1. The patients in the nonbiologics group were significantly older than those in the biologics group (72.5 versus 66.4 years, $P=0.045$ ), but no significant differences were found in smoking history $(33.3 \%$ versus $41.7 \%, P=1.00)$, duration from onset of symptoms to PCP diagnosis (7.7 versus 11.1 days, $P=0.44$ ), comorbidities, and concomitant immunosuppressive treatments, between the biologics and nonbiologics groups.

While the biologics group had significantly lower serum levels of $\beta$-D-glucan (100 versus $231 \mathrm{pg} / \mathrm{mL}, P=0.039$ ) and CRP (5.2 versus $10.6 \mathrm{mg} / \mathrm{dL}, P=0.039)$ than the nonbiologics group, the biologics group had significantly higher lymphocyte counts than the nonbiologics group (1429 versus 585 cells $/ \mu \mathrm{L}, P=0.033$ ), implying that patients on biologics develop PCP with almost normal lymphocyte counts accompanied with low $\beta$-D-glucan levels. No significant differences were found in total white blood cell counts (5358 versus 7217 cells/ $\mu \mathrm{L}, P=0.51$ ), the levels of IgG (1127 versus 896 cells $/ \mu \mathrm{L}, P=0.26)$, LDH (298 versus $389 \mathrm{U} / \mathrm{L}$, $P=0.11)$, KL-6 (632 versus $1008 \mathrm{U} / \mathrm{mL}, P=0.22)$, and the $\mathrm{PaO}_{2} / \mathrm{FiO}_{2}$ ratio (293 versus $281, P=0.53$ ). The survival rate was not different in both groups (91.7\% versus $83.3 \%, P$ $=1.00)$.

3.3. Factors Associated with the Severity of PCP. Furthermore, we analysed the relationship between the $\mathrm{PaO}_{2} / \mathrm{FiO}_{2}$ ratio, which is used to assess the severity of pneumonitis, and laboratory findings. In all the patients $(n=24)$, serum levels of CRP and LDH negatively correlated with the $\mathrm{PaO}_{2} / \mathrm{FiO}_{2}$ ratio, while lymphocyte counts and serum albumin levels positively correlated with the $\mathrm{PaO}_{2} / \mathrm{FiO}_{2}$ ratio (Figure 2(a)). However, when we performed the same analysis separately in the biologics group and in the nonbiologics group, only the CRP levels negatively correlated with the $\mathrm{PaO}_{2} / \mathrm{FiO}_{2}$ ratio in both groups (Figure 2(b)). The significant correlation with the other variables, except for $\mathrm{LDH}$ in the nonbiologics group, was no longer detected in either group, suggesting that the CRP level was the most important index for assessing the severity of PCP. 
TABLE 1: Comparison of demographic characteristics of RA-PCP at diagnosis.

\begin{tabular}{|c|c|c|c|c|}
\hline Characteristics & $\begin{array}{c}\text { All patients } \\
N=24\end{array}$ & $\begin{array}{c}\text { Biologics group } \\
\quad N=12\end{array}$ & $\begin{array}{c}\text { Nonbiologics group } \\
\quad N=12\end{array}$ & Pvalue ${ }^{*}$ \\
\hline Age, years, mean (SEM) & $69.5(1.5)$ & $66.4(2.2)$ & $72.5(1.7)$ & 0.045 \\
\hline Female, $n(\%)$ & $20 / 24(83.3)$ & $10 / 12(83.3)$ & $10 / 12(83.3)$ & 1.00 \\
\hline Smoking, $n(\%)$ & 9/24 (56.3) & $4 / 12(33.3)$ & $5 / 12(41.7)$ & 1.00 \\
\hline Disease duration, days, mean (SEM) & $9.4(2.2)$ & $7.7(1.0)$ & $11.1(4.2)$ & 0.44 \\
\hline \multicolumn{5}{|l|}{ Comorbidity, $n(\%)$} \\
\hline Renal dysfunction ${ }^{\ddagger}$ & $13 / 24(54.2)$ & $6 / 12(50.0)$ & $7 / 12(58.3)$ & 1.00 \\
\hline Lung disease $e^{\ddagger \ddagger}$ & $10 / 24(41.7)$ & $4 / 12(33.3)$ & $6 / 12(50.0)$ & 0.68 \\
\hline Heart failure & $3 / 24(12.5)$ & $1 / 12(8.3)$ & $2 / 12(16.7)$ & 1.00 \\
\hline Liver dysf unction ${ }^{\ddagger \ddagger}$ & $10 / 24(41.7)$ & $4 / 12(33.3)$ & $6 / 12(50.0)$ & 0.68 \\
\hline Diabetes mellitus & $7 / 24(29.2)$ & $3 / 12(25.0)$ & $4 / 12(33.3)$ & 0.67 \\
\hline \multicolumn{5}{|l|}{ Concomitant treatment, $n(\%)$} \\
\hline Glucocorticoid & $17 / 24(70.8)$ & $7 / 12(58.3)$ & $10 / 12(83.3)$ & 0.37 \\
\hline Dose of glucocorticoid (mg/day), mean (SEM) & $7.7(1.7)$ & $5.7(1.1)$ & $9.1(2.7)$ & 0.60 \\
\hline Methotrexate & $23 / 24(95.8)$ & $11 / 12(91.7)$ & $12 / 12(100.0)$ & 1.00 \\
\hline Dose of methotrexate (mg/week), mean (SEM) & $9.2(0.6)$ & $8.9(0.6)$ & $9.5(0.9)$ & 0.93 \\
\hline \multicolumn{5}{|l|}{ Laboratory findings } \\
\hline $\mathrm{PaO}_{2} / \mathrm{FiO}_{2}$ ratio, mean $(\mathrm{SEM})$ & $287(20)$ & $293(33)$ & $281(25)$ & 0.53 \\
\hline White blood cells $(/ \mu \mathrm{L})$, mean (SEM) & $6288(700)$ & $5358(732)$ & $7217(1165)$ & 0.51 \\
\hline Lymphocytes $(/ \mu \mathrm{L})$, mean (SEM) & $1007(201)$ & $1429(357)$ & $585(96)$ & 0.033 \\
\hline Albumin, g/dL, mean (SEM) & $3.0(0.1)$ & $3.3(0.2)$ & $2.7(0.2)$ & 0.06 \\
\hline CRP, mg/dL, mean (SEM) & $7.9(1.2)$ & $5.2(1.7)$ & $10.6(1.5)$ & 0.039 \\
\hline IgG, mg/dL, mean (SEM) & $1023(91)$ & $1127(124)$ & $896(128)$ & 0.26 \\
\hline LDH, U/L, mean (SEM) & $343(24)$ & $298(24)$ & $389(38)$ & 0.11 \\
\hline KL-6, U/mL, mean (SEM) & $793(117)$ & $632(100)$ & $1008(226)$ & 0.22 \\
\hline$\beta$-D glucan, pg/mL, mean (SEM) & $166(32)$ & $100(30)$ & $231(52)$ & 0.039 \\
\hline Death, $n(\%)$ & $3 / 24(12.5)$ & $1 / 12(8.3)$ & $2 / 12(16.7)$ & 1.00 \\
\hline
\end{tabular}

${ }^{*}$ Comparison between biologics and nonbiologics group. ${ }^{\ddagger}$ Renal dysfunction: estimated glomerular filtration rate under $60 \mathrm{~mL} / \mathrm{min} / 1.73 \mathrm{~m}{ }^{2} .{ }^{\ddagger \ddagger} \mathrm{Lung}$ disease: interstitial lung disease, bronchiolitis, and chronic obstructive pulmonary disease. ${ }^{\ddagger \ddagger \ddagger}$ Liver dysfunction: alanine aminotransferase over 37 IU/L. Biologic agents: infliximab, etanercept, adalimumab, golimumab, and abatacept. RA, rheumatoid arthritis; PCP, pneumocystis pneumonia; LDH, lactate dehydrogenase; CRP, C-reactive protein; KL-6, Krebs von den Lungen-6.

3.4. The Association of Lymphocyte Count with $\beta$-D-Glucan Levels. We analysed the relationship between the three parameters that differed between the biologics and nonbiologics groups (lymphocyte count, $\beta$-D-glucan, and CRP). In the nonbiologics group, lower lymphocyte counts were associated with higher $\beta$-D-glucan levels (Figure 3), implying that the compromised immune system of patients in the nonbiologics group was related to the growth of Pneumocystis jirovecii. Interestingly, this relationship was not found in the biologics group (Figure 3). CRP levels were not related to either lymphocyte counts or $\beta$-D-glucan levels in either group.

Further on, we performed the same analysis on the 16 patients with definite PCP (the biologics group, $n=7$; the nonbiologics group, $n=9$ ). Likewise, lower lymphocyte counts were associated with higher $\beta$-D-glucan levels only in the nonbiologics group $(\rho=-0.683, P=0.042)$, while this association was not observed in the biologics group ( $\rho$ $=0.679, P=0.094)$.

\section{Discussion}

In this study, we revealed that, in patients with RA who are being treated with biologic agents, PCP developed with almost normal lymphocyte counts and lower $\beta$-D-glucan levels, whereas in those being treated with nonbiologic DMARDs, PCP was marked by high $\beta$-D-glucan levels with apparent lymphocytopenia. The findings suggest that the pathophysiology of PCP in patients being treated with biologic agents is different from that in patients being treated with nonbiologic DMARDs. Furthermore, we found that only serum CRP levels, but not $\beta$-D-glucan levels, reflected the severity of PCP in patients with RA.

Assuming that $\beta$-D-glucan levels indicate the growth of Pneumocystis jirovecii and lymphocyte counts represent the degree of compromise on the immune system, the differences in the burden of Pneumocystis jirovecii and immunosuppression in our study suggest that the mechanism of PCP development with the use of biologic agents is substantially 

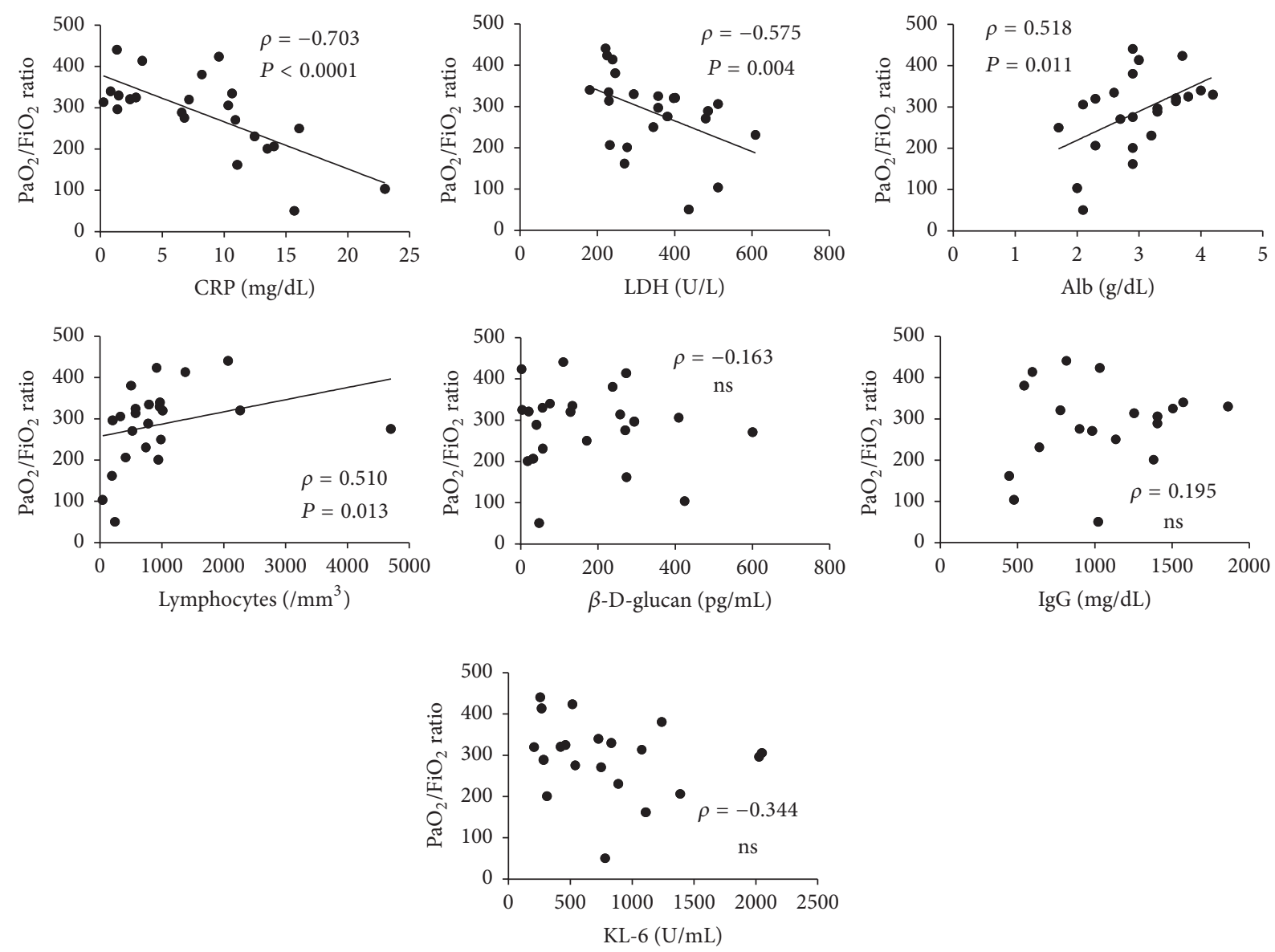

(a)
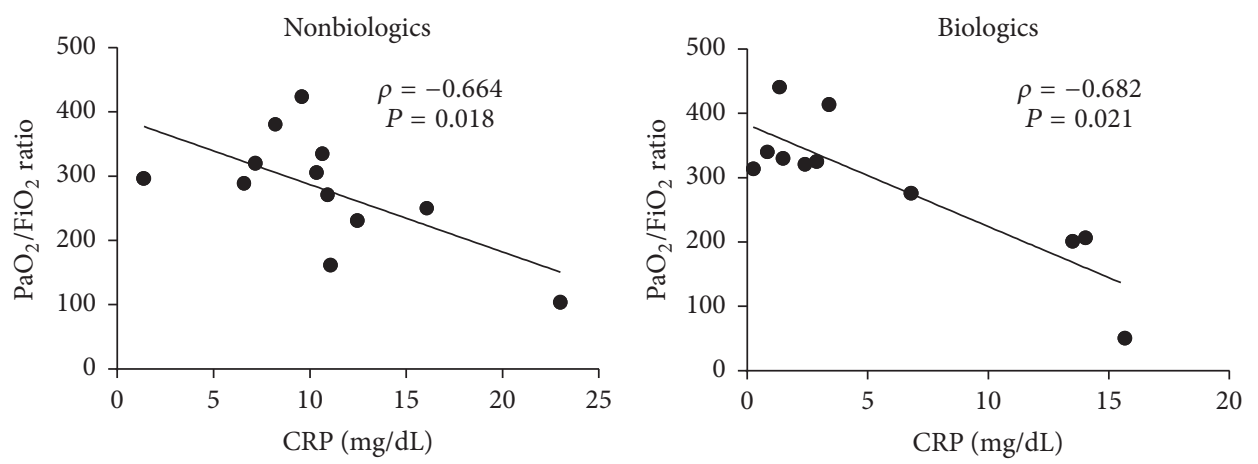

(b)

FIgure 2: The association of $\mathrm{PaO}_{2} / \mathrm{FiO}_{2}$ ratio with laboratory findings. (a) Total PCP patients. (b) The biologics group and the nonbiologics group. CRP, C-reactive protein. LDH, lactate dehydrogenase. KL-6, Krebs von den Lungen-6. NS, not significant.

different from that with the use of nonbiologic DMARDs. A previous study reported that alveolar macrophages are crucial in mediating the clearance of Pneumocystis jirovecii from the lungs [11], through the production of a large amount of tumour necrosis factor- $\alpha$ (TNF- $\alpha$ ) [12]. An experiment on murine models of PCP proved that the clearance of Pneumocystis jirovecii infection was impaired when TNF- $\alpha$ was neutralized by TNF- $\alpha$ inhibitors [13]. Thus, PCP development during the use of biologic agents, which directly inhibit cytokines such as TNF- $\alpha$, may be caused by impaired primary host immune responses of alveolar macrophages, rather than by lymphocyte suppression. Conversely, the pathogenesis of PCP during the use of nonbiologic DMARDs may be ascribed to immunosuppression that manifests with lymphocytopenia. In fact, lymphocytes play a crucial role in host defence against Pneumocystis jirovecii, both in humans and in murine models, with an increased risk of PCP development in patients with markedly decreased lymphocyte counts $[14,15]$. Consistent with these reports, our study showed that there is a significant correlation between lower lymphocyte counts and 

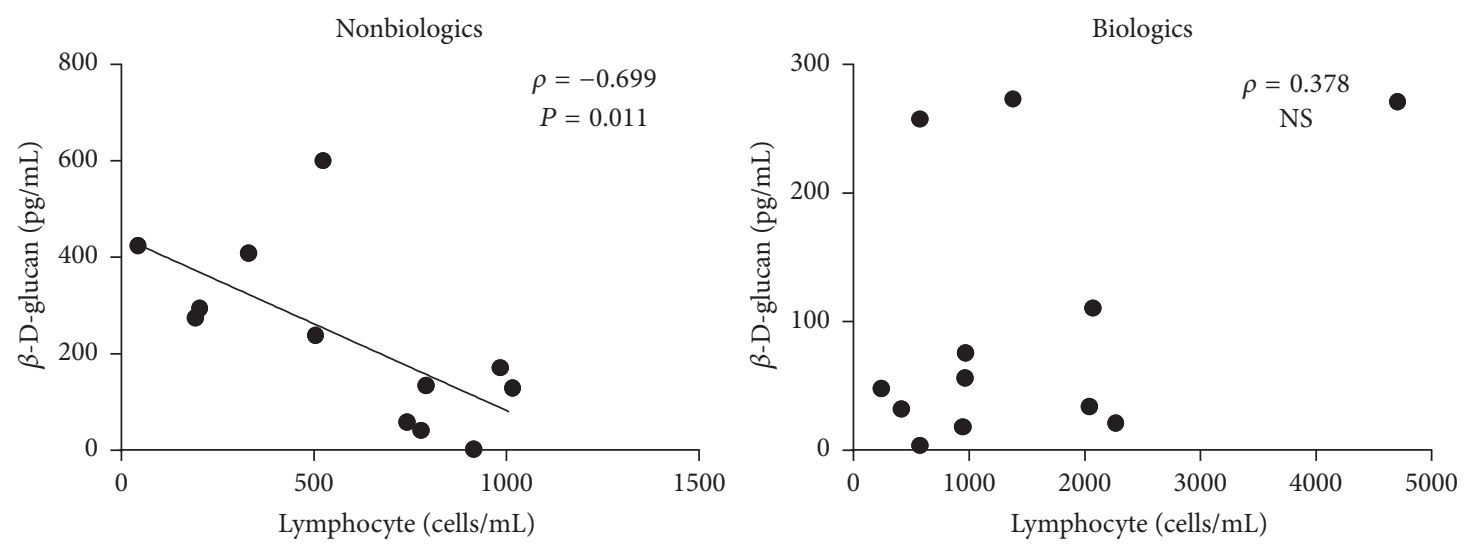

FIGURE 3: The association of lymphocyte counts with serum levels of $\beta$-D-glucan. NS, not significant.

the burden of Pneumocystis jirovecii, indicated by serum $\beta$ D-glucan levels in patients receiving nonbiologic DMARDs.

Respiratory impairment is more closely associated with the extent of lung inflammation than with the amount of Pneumocystis jirovecii burden in HIV-infected patients, and the same finding was reported in non-HIV-infected patients [16]. Similarly, our present study showed that respiratory impairment, represented by the $\mathrm{PaO}_{2} / \mathrm{FiO}_{2}$ ratio, was related to the levels of serum CRP, but not to the levels of serum $\beta$ $\mathrm{D}$-glucan in both the biologics and the nonbiologics groups. PCP severity may be a result of lung inflammation, disturbing gas exchange, and may be unaffected by the quantity of Pneumocystis jirovecii.

We note that our study has limitations. This is a retrospective, observational study with a small sample size, although the initial sample of patients with RA, with or without PCP, was of a large number. Also, there is a little possibility that some lost-to-follow-up patients may have developed PCP; however, we believe they would be very few considering the low incidence of PCP and would not change the results of our study. We believe that our present study is important for increasing the understanding of this rare, critical complication of PCP in patients with RA being treated with biologic agents. Further prospective studies in larger cohorts of patients are needed to confirm our results.

In conclusion, in patients with RA being treated with biologic agents, PCP develops with nearly normal lymphocyte counts and low $\beta$-D-glucan levels. Rheumatologists should recognize these newly identified characteristics of this rare but critical complication during treatment with biologic agents to ensure early diagnosis and appropriate treatment.

\section{Conflicts of Interest}

Mitsuhiro Akiyama has received consultancies, speaking fees, and honoraria from Cure Grades Co. and Eisai Co., Ltd. Yuko Kaneko received consulting fees, speaking fees, and/or honoraria from AbbVie, Astellas Pharma, Chugai Pharmaceutical, Bristol-Myers K.K., Eisai, Mitsubishi Tanabe Pharma, Pfizer Japan, and Takeda Pharmaceutical (less than $\$ 10,000$ each). Tsutomu Takeuchi received consulting fees, speaking fees, and/or honoraria from Pfizer Japan, Mitsubishi Tanabe Pharma, Eisai, Astellas Pharma, and UCB (less than $\$ 10,000$ each) and from Chugai Pharmaceutical, BristolMyers K.K., Daiichi Sankyo, AbbVie, Janssen Pharmaceutical K.K., Pfizer Japan, Asahi Kasei Pharma, Takeda Pharmaceutical, AstraZeneca K.K., Eli Lilly Japan K.K., and Novartis Pharma K.K. (more than $\$ 10,000$ each).

\section{Acknowledgments}

The authors sincerely thank all the physicians and others who provided care for the patients enrolled in this study.

\section{References}

[1] S. Tasaka and H. Tokuda, "Pneumocystis jirovecii pneumonia in non-HIV-infected patients in the era of novel immunosuppressive therapies," Journal of Infection and Chemotherapy, vol. 18, no. 6, pp. 793-806, 2012.

[2] R. M. Selik, E. T. Starcher, and J. W. Curran, "Opportunistic diseases reported in AIDS patients: frequencies, associations, and trends," AIDS, vol. 1, no. 3, pp. 175-182, 1987.

[3] Y. Kaneko, A. Suwa, Y. Ikeda, and etal., "Pneumocystis jiroveci pneumonia associated with low-dose methotrexate treatment for rheumatoid arthritis: report of two cases and review of the literature," Modern Rheumatology, vol. 16, no. 1, pp. 36-38, 2006.

[4] S. Mori and M. Sugimoto, "Pneumocystis jirovecii infection: an emerging threat to patients with rheumatoid arthritis," Rheumatology, vol. 51, no. 12, pp. 2120-2130, 2012.

[5] E. S. Bruce, L. Kearsley-Fleet, K. D. Watson, and etal., "Risk of Pneumocystis jirovecii pneumonia in patients with rheumatoid arthritis treated with inhibitors of tumour necrosis factor $\alpha$ : results from the british society for rheumatology Biologics Register for Rheumatoid Arthritis," Rheumatology, vol. 55, no. 7, pp. 1336-1337, 2016.

[6] M. Tanaka, R. Sakai, R. Koike, and M. Harigai, "Pneumocystis jirovecii pneumonia in Japanese patients with rheumatoid arthritis treated with tumor necrosis factor inhibitors: a pooled analysis of 3 agents," Journal of Rheumatology, vol. 42, no. 9, pp. 1726-1728, 2015.

[7] P. D. Walzer, M. LaBine, T. J. Redington, and etal., "Lymphocyte changes during chronic administration of and withdrawal from 
corticosteroids: Relation to Pneumocystis carinii pneumonia," Journal of Immunology, vol. 133, no. 5, pp. 2502-2508, 1984.

[8] Y. Komano, M. Harigai, and R. Koike, "Pneumocystis jiroveci pneumonia in patients with rheumatoid arthritis treated with infliximab: a retrospective review and case-control study of 21 patients," Arthritis Rheumatism, vol. 61, no. 3, pp. 305-12, 2009.

[9] H. Kameda, H. Tokuda, F. Sakai, and etal., "Clinical and radiological features of acute-onset diffuse interstitial lung diseases in patients with rheumatoid arthritis receiving treatment with biological agents: importance of pneumocystis pneumonia in japan revealed by a multicenter study," Internal Medicine, vol. 50, no. 4, pp. 305-313, 2011.

[10] S. Tasaka, N. Hasegawa, S. Kobayashi, and etal., "Serum indicators for the diagnosis of pneumocystis pneumonia," Chest, vol. 131, no. 4, pp. 1173-1180, 2007.

[11] C. F. Thomas Jr. and A. H. Limper, "Current insights into the biology and pathogenesis of Pneumocystis pneumonia," Nature Reviews Microbiology, vol. 5, no. 4, pp. 298-308, 2007.

[12] L. W. Neese, J. E. Standing, E. J. Olson, and etal., "Vitronectin, fibronectin, and gp120 antibody enhance macrophage release of TNF- $\alpha$ in response to Pneumocystis carinii," Journal of Immunology, vol. 152, no. 9, pp. 4549-4556, 1994.

[13] T. W. Wright, G. S. Pryhuber, P. R. Chess, and etal., "TNF Receptor Signaling Contributes to Chemokine Secretion, Inflammation, and Respiratory Deficits during Pneumocystis Pneumonia," Journal of Immunology, vol. 172, no. 4, pp. 2511-2521, 2004.

[14] J. Phair, A. Muñoz, R. Detels, and etal., "The risk of Pneumocystis carinii pneumonia among men infected with human immunodeficiency virus type 1. Multicenter AIDS Cohort Study Group," New England Journal of Medicine, vol. 322, no. 3, pp. 161-165, 1990.

[15] J. Shellito, V. V. Suzara, W. Blumenfeld, and etal., "A new model of Pneumocystis carinii infection in mice selectively depleted of helper T lymphocytes," Journal of Clinical Investigation, vol. 85, no. 5, pp. 1686-1693, 1990.

[16] A. H. Limper, K. P. Offord, T. F. Smith, and etal., "Pneumocystis carinii pneumonia. Differences in lung parasite number and inflammation in patients with and without AIDS," American Review of Respiratory Disease, vol. 140, no. 5, pp. 1204-1209, 1989. 


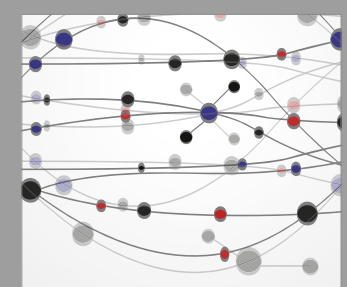

The Scientific World Journal
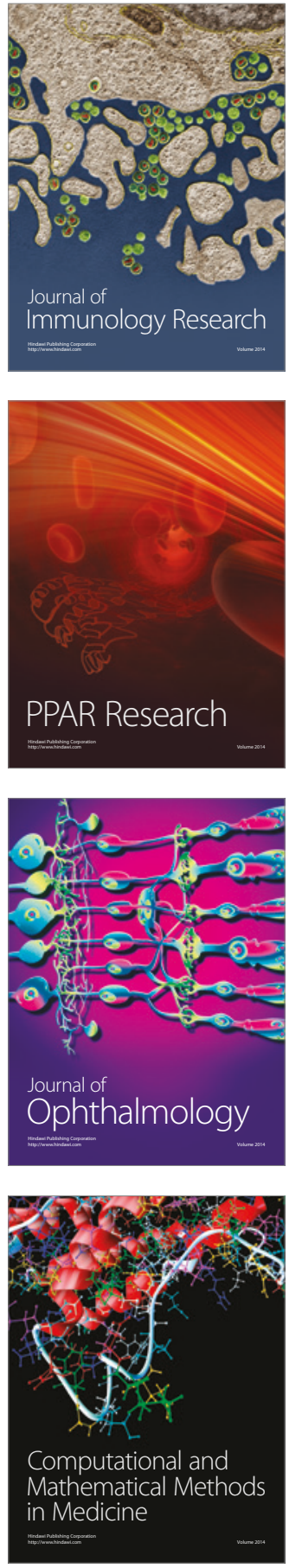

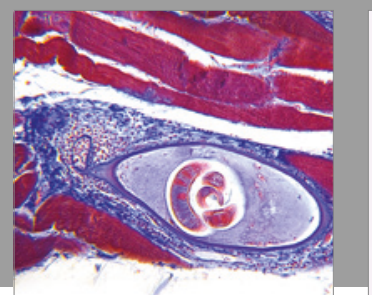

Gastroenterology Research and Practice
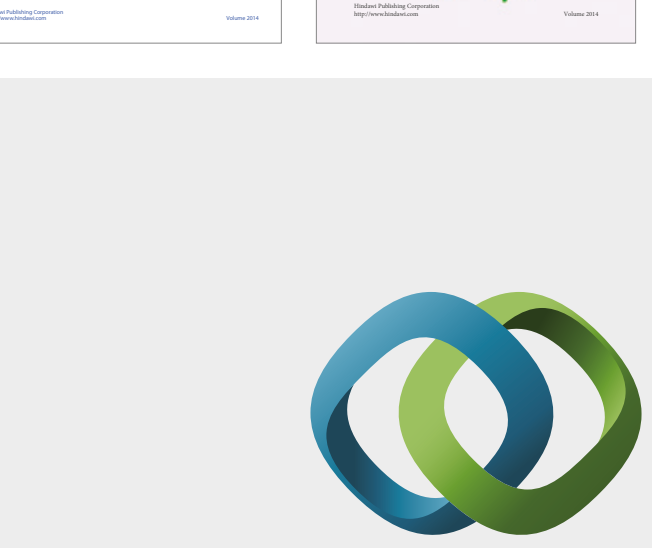

\section{Hindawi}

Submit your manuscripts at

https://www.hindawi.com
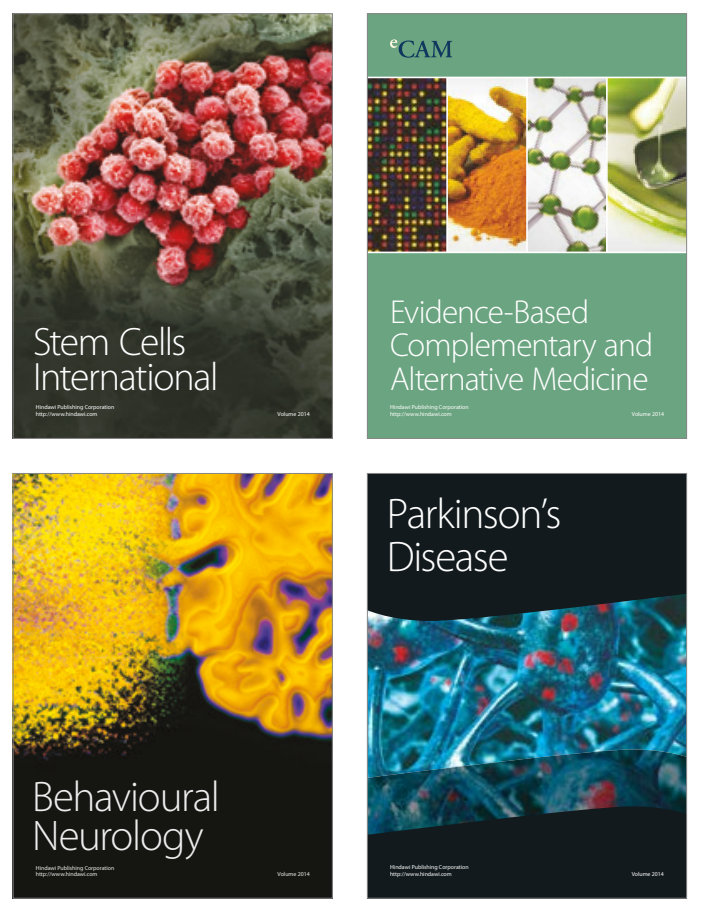
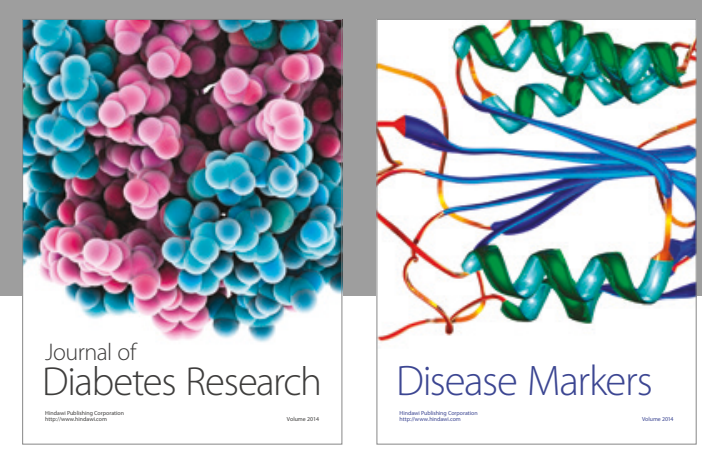

Disease Markers
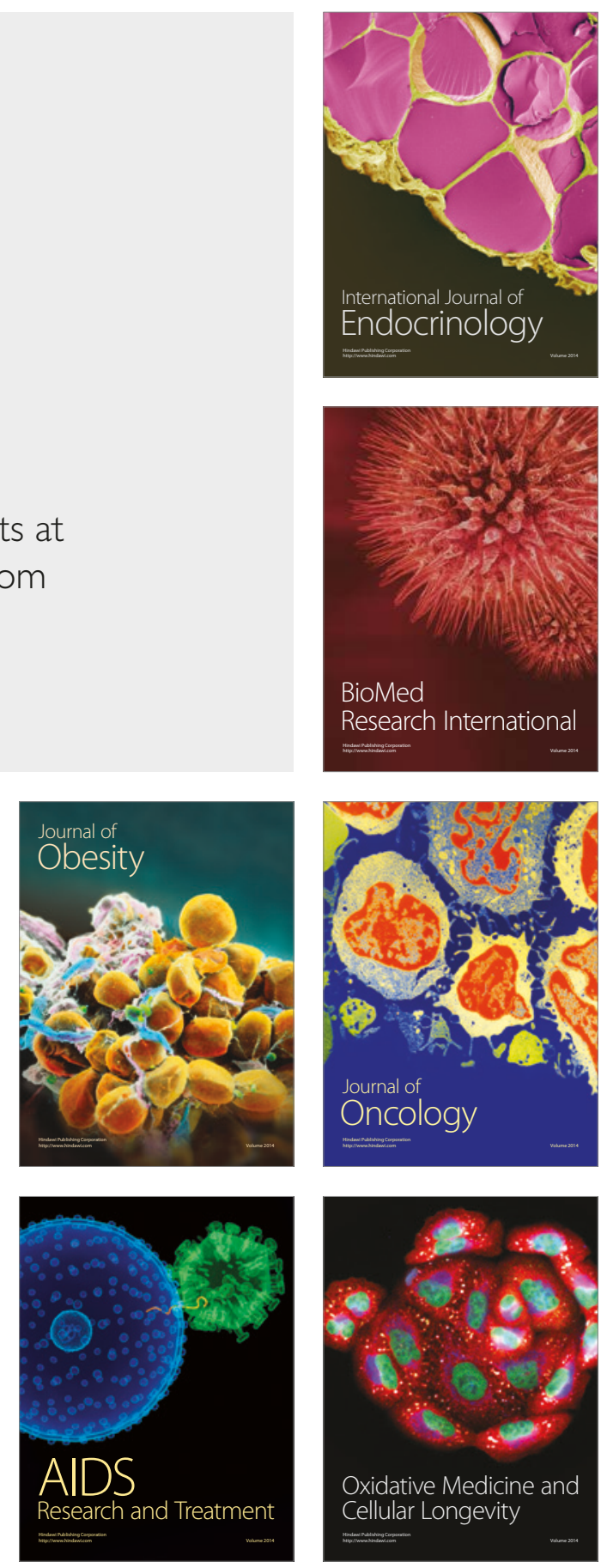